\title{
The conceptual framework for physical risk assessment in multi-purpose workplaces
}

\author{
Andrzej Marek Lasota ${ }^{1, *}$ and Krzysztof Hankiewicz ${ }^{2}$ \\ ${ }^{1}$ University of Zielona Gora, Institute of Safety Engineering \& Work Science, 4 Prof. Z.Szafrana \\ Street, 65-516 Zielona Gora, Poland \\ ${ }^{2}$ Poznan University of Technology, Chair of Management and Computing Systems, 11 Strzelecka \\ Street, 61-845 Poznan, Poland
}

\begin{abstract}
In industry work related musculoskeletal disorders (WRMSDs) are still a common problem which frequent cause of health problems, sick leave and it can result in decreased productivity, quality of work and increased absenteeism. Though, traditional manufacturing work practices do not enough take into account task variability issues during the work design - assessment process. Variations in task content and organizational work performance are due to effective use of equipments and reaching high level of productivity. But this variation has impact on work demand and risk to WRMSDs and makes some difficulties with assessment of risk. Therefore, this study aims develop a framework for assessment multipurpose workplaces. The proposed method integrates complementary concepts of widely known techniques used for evaluation of physical risk factors to WRMSDs on workplaces. Additionally the research framework is highlighting major differences in worker's exposure to WRMSDs risk, potential impact on awkward postures, and how these findings can be used for finding solutions in a future ergonomic intervention.
\end{abstract}

\section{Introduction}

For many years, the importance of ergonomics and human factors has grown in the industry. Ergonomic work environment is directly dependent on design and ergonomic evaluation process [1]. Ergonomic evaluation is inextricably linked to work safety and workplace risk. Taking into account safety aspects the machine design and construction phases significantly determines reduction of risks on work stands [2]. It is a well-known fact that stress also determines risk. However, the results showed that workers experience workplace stress associated with the functions of the work environment in terms of work organization more often than job content stressors related to the type of tasks [3]. Additionally quality management also define work environment [4].

Employee productivity depends, among other resulting from the Work-related Musculoskeletal Disorders (WRMSDs), which cause health problems, sick leave and may reduce the quality of work, increasing cost and absenteeism in many occupations [5].

\footnotetext{
*Corresponding author: am.lasota@o2.pl
} 
Moreover, the growing number of older workers can change statistic values and causes the need to design workplaces for these employees as well [6].

To avoid WRMSDs, musculoskeletal load must be on appropriate level and researchers have developed a wide range of methods to estimate the risk to WRMSDs: Ovako Working Posture Analysing System (OWAS) [7], Rapid Upper Limb Assessment [8], Rapid Entire Body Assessment [9], Occupational Repetitive Action [10] and Quick Exposure Check [11]. These methods are widely used in various industries [12-15].

In the scientific literature, there are plenty of studies reported health outcomes and assessment of risk to WRMSDs of occupational groups, at production and assembly process [12] used mentioned risk assessment method to evaluate jobs, but many industrial jobs are multi-task in nature [16] and there are multi-purpose workplaces. Moreover, the growing level of customization and shorter product life manifests itself in the form of smaller batch sizes. Such production system results in that on the same machines, workplaces variety of components are manufactured after retooling/rearrangement. This diversity results in variability of tasks performed by operators which could have an influence on the workload and posture of an operator during work, repetition, excessive physical load, duration of movement and finally the risk of WRMSDs. Variability of tasks on the multi-purpose workplace increase the difficulty associated with estimating the ergonomic risk and with identifying reasons of risk which in turn makes it difficult to formulate recommendations and take corrective action. Therefore, the objective of this study was to develop a framework of physical risk assessment on multi-purpose workplaces.

\section{Research method}

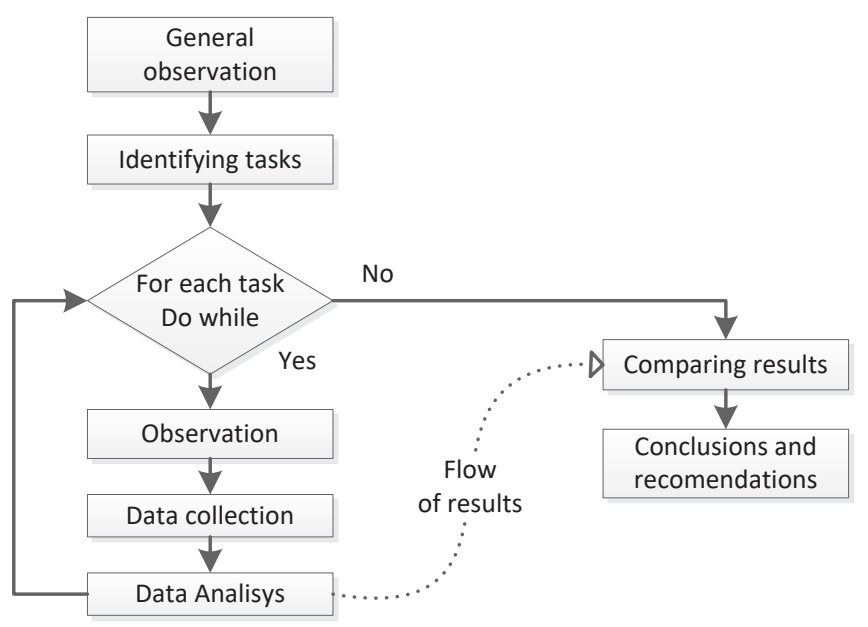

Fig. 1. Flowchart of the proposed framework

The proposed research method is a framework (Figure 1). To reach the objectives of this study, the research method began with general observation. This initial step supplies insights about job performed on a chosen workplace. After that, the identification of tasks on entire workplace is possible and evaluation of each task in based on three main steps: 1) observation of single task, 2) data collection, and 3) data analyzing.

On the next stage the results from all assessed tasks are correlated for comparison and analyses. This makes it possible to formulate conclusions and recommendations for identified reasons of abnormalities and risk, and improvement opportunities. 


\subsection{General observation}

The purpose of this step is general observation of multi-purpose workplace for assessment in the field were multi-task is done on a single workplace. The general visual observation and interview with managers, supervisors and technologists could be helpful to understand of multi-purpose workplace. The collected information from supervisors should help to understand job and work activities performed by operator(s).

\subsection{Identifying tasks}

Observation employee(s) during their daily routine work and in the current and real working environment, group discussion and interview should be used to identify tasks performed on the chosen workplace. It could help establish the priority and sequence of assessment, data collection method(s), assessment strategy.

\subsection{For each task}

For each identified task performed on the selected workplace it should be done observation, data collection, and data analysis.

\subsubsection{Observation}

Observation employee during their daily routine work in the current task, real working environment, and interview with him/her should be used to explore any problems and difficulties in performing task. It also should helps in creating a friendly atmosphere for carrying research.

\subsubsection{Data collection}

Several methods of data collection are possible: observation, interview, task analysis. Visual observation is very simple and useful, but video recording has many advantages. It allows carrying out analysis after the event and it could be repeated many times and does not interference with work patterns. Tasks analyses and interview will depend on the objectives of the study and method(s) of evaluation.

\subsubsection{Data analysis}

This step is an in-depth analysis of all data collected. Analysis of risk factors and the level of risk to WRMSDs can be made through defined ergonomic evaluation criteria and WRMSDs evaluation method(s).

\subsection{Comparison of results}

The results from the observational study for each task indicate the levels of risk involved with the adopted working postures and additional physical factors e.g. repetitions, load/force and the final action categories. They provide additional information about workload, repetitiveness and awkward body segment posture for each task. The level of action category gives key information and guidelines to the observer which adopted working postures by workers are harmful, and what level of urgency of ergonomic intervention is required. Summary of the results of all assessed tasks on evaluated 
workplace provides to the observer extra information about the awkward postures, action categories and activities to which they relate. A comparison of results allows determining during which activities the employee adopting incorrect postures and in what part of these activities are common to all assessed tasks. This information is necessary to propose solutions; especially the solution focuses on changes in the workplace.

\subsection{Conclusions and recommendations}

In this step after identifying abnormalities e.g. vibration, awkward working postures, it is straightforward to conclude about WRMSDs risk in each task and about the reasons of harmful action categories and non neutral body segment postures. Furthermore, these conclusions have to be taken as a recommendation for improving working condition. It should further be used with fundamental ergonomics principles to develop of corrective actions and ergonomic solutions which will take into consideration demands flowing from all tasks performed on assessed workplace. Additionally it should help in formulation solution fulfilling all demands flowing from all assessed tasks.

\section{The case study in a fittings factory}

The study was conducted at a workplace of manual processing of rubber components. The operator aged 51 years, 3 years experience manually treated: rubber feet, rubber elbows, and compensators, with the use of: scissors, scalpel or knife. She manually cut out rubber surpluses that flowed from the mold under pressure from a hydraulic press.

\subsection{General observation}

The general observation of multi-purpose workplace in the field, face-to-face interview with supervisor and collected information from worker and supervisor helped to understand job and work activities performed by operator.

\subsection{Identifying tasks}

Observation employee in the field, discussion and previous information was used. A strategy of assessment was developed. Authors' used pen and paper observation method with sampling (interval - 25 seconds). The OWAS method as a one of the simpler technique which allows on quick assessment was used. It was identified three tasks performed on the workplace: Task\#1: processing of rubber feet. The employee reaching the container from the cart, then empty out parts on the table top, empty container put off on the side, cut off rubber surpluses from parts, and then ready parts put off into a container. After graduating from the party put off a container on a cart. Task\#2: processing of rubber compensators. She was reaching for two parts from the container located on the cart (one piece in left hand, second piece in right hand), and then put on the table, cut off surplus of rubber, and then put down the finished parts on the second cart. Task\#3: processing of rubber elbows. A worker reached a one piece with both hands from the palette, has put on the table top, cut off surplus of rubber, and then put down the finished parts on the pallet.

\subsection{For each task}

For each of three identified task performed on the selected workplace it was done observation, data collection, data analysis. 


\subsubsection{Observation}

Observation employee was done during their daily routine work in the current task and real working environment, and interview with her. She was asked about task performed at the workplace. It allowed get more information about tasks; explore problems and difficulties on assessed task, job and workplace.

\subsubsection{Data collection}

Data collection consisted of recording the postures through visual observation. The evaluations were recorded for each task separately. In total 212 work postures were evaluated: 48 for task\#1, 75 - task\#2 and 89 - task\#3. The OWAS method was used. It is based on a classification of different positions for the back, arms, legs and the force/load used in the work. It uses a four-digit code to describe various postures and force/load combinations. These four-digit codes from different body parts in a specific position combined with the estimated load provide information about each postural load. The combination creates categories describing the risk of exposure to WRMSDs and action categories (AC) necessary to improve the working conditions: AC 1 - no risk, intervention is not required; AC 2 - low risk, immediate intervention is not required, but the ergonomic adjustment should be taken into account in future actions; AC 3 - medium risk, ergonomic intervention should be carried out as soon as possible; AC 4 - high risk, ergonomic intervention is required immediately.

\subsubsection{Data analysis}

The recorded data were separately studied for each task. Four digit code for each recorded postures and action categories included harmless (AC1) and harmful (AC 2, AC 3, AC4). For task\#1, authors' findings show that the most common four-digit posture code was: 1111 - 35.4\%, classified to AC1; 2111 (33.4\%, AC2) and $3211(25.0 \%$, AC2). The $65 \%$ of postures were classified to harmful action categories. In the case of task\#2 the most common four-digit posture code: $2111,64.0 \%$ and classified to AC2, and 1211, 16.0\%, $\mathrm{AC} 1$. The $76 \%$ of postures were classified to harmful action categories. For task\#3 2131 $36 \%, \mathrm{AC} 2 ; 2231-23.6 \%, \mathrm{AC} 2$ and $1121,13.5 \%$, AC1 were the most common four-digit postures. The $72 \%$ of postures were classified to harmful action categories.

\subsection{Comparison of results}

This step shows that the most common four digit code posture were $2111,30.2 \%, \mathrm{AC} 2$ and $2131,15.1 \%$, AC2. The $72 \%$ of postures were classified to harmful action categories. In total, two postures classified to AC4: one 4141 (reaching for two parts from the container located on the cart) at task\#2 and one 4151 (putting the container with ready parts on the cart) at task\#1. Six postures classified to AC3: one posture coded 2151, which occurred at task\#1 (reaching of container with parts from the cart); two postures: 2152 at task\#3 (reaching a part from the pallet located on the floor); two postures: 2172 occurred at task\#3 (transferring a part from the palette to the workstation); and one postures 2251 at task\#2 (putting the part in the container positioned on the cart).

The comparison in this study shows that posture classified to AC2 and coded as: 2111, 2121, 2131, 2211 and 3211 identified on two different tasks. 


\subsection{Conclusions and recommendations}

The results indicate differences in the $\mathrm{AC}$ for each evaluated task and incorrect working postures. The common problem for all evaluated tasks for this workplace is reaching in and laying on the cart or the pallet. This fact requires common solution.

\section{Conclusions}

The proposed framework allows identifying the physical risk factors of the multi-purpose workplace created with the each task and whole job performed on the workplace supporting improvement actions. It is based on traditional assessment method included pen and papers.

Further researches are necessary to improve efficiency of using framework. It will be focused to confirm validity and reliability of proposed method and it can be two-faceted. Firstly, in the design process - e.g. in stage of simulation in virtual environment will use digital human modeling for ergonomic assessments proposed solutions. Secondly, in the field will use on different type of multi-purpose workplaces.

\section{References}

1. A. Górny, Procedia Manufacturing, 3, 4700 (2015)

2. B. Mrugalska, P. M. Arezes, Occupational Safety and Hygiene, 97 (2013)

3. A. Mościcka-Teske, J. Sadłowska-Wrzesińska, M. Butlewski, A. Misztal, A. Jacukowicz, Int J Occup Saf Ergo, 1 (2016)

4. A. Górny, The Shaping of Work Environment, C. Stephanidis (ed.), Posters Extended Abstracts: International Conference, HCI International 2015 (part II), Communications in Computer and Information Science, 529, 136 (2015)

5. BLS, Bureau of Labor Statistics 2016, Nonfatal Occupational Injuries and Illnesses Requiring Days Away from Work, http://www.bls.gov/news.release/pdf/osh2.pdf (2016)

6. M. Butlewski, Applied Mechanics and Materials 657, 1061 (2014)

7. O. Karhu, P. Kansi, I. Kuorinka, Appl Ergon, 8 (4), 199 (1977)

8. L. McAtamney, E. N. Corlett, Appl Ergon, 24 (2), 91 (1993)

9. S. Hignett, L. McAtamney, Appl Ergon, 31 (2), 201 (2000)

10. E. Occhipinti, D. Colombini, Med Lav, 87 (6), 491 (1996)

11. G. David, V. Woods, P. Buckle, Appl Ergon, 39 (1), 57 (2008)

12. A. M. Lasota, LogForum, 10 (4), 383 (2014)

13. A. M. Lasota, K. Hankiewicz, Evaluation of ergonomic risk in the production line of frozen food products, in: Yue, X.G., Duarte, N.J.R. (Eds.), International Conference on Economics and Management Innovations - CEMI, ACSR-Advances in Computer Science Research. Atlantis Press, Paris, France, 272 (2016)

14. A. M. Lasota, K. Hankiewicz, International Symposium on Occupational Safety and Hygiene - SHO 2016; Proceedings book. Guimaraes, Portugal, 138-140 (2016)

15. A. M. Lasota, K. Hankiewicz, Occupational safety and hygiene IV, Taylor \& Francis Group, London, 261 (2016)

16. P. Drinkaus, R. Sesek, D. S. Bloswick, C. Mann, T. Bernard, Int J Occup Saf Ergo, 11(3), 263-281 (2005) 